\title{
Consumo de metal durante la Prehistoria Reciente en el centro de la Península Ibérica. Una aproximación a través del análisis de los contextos funerarios en fosa
}

\author{
Metal consumption in the Late Prehistory of the central Iberian Peninsula: an approach \\ through the analysis of pit grave contexts
}

\author{
Eduardo Carmona Ballestero (*) \\ Miguel Ángel Arnaiz Alonso (*) \\ Juan Montero Gutiérrez (*)
}

\section{RESUMEN}

El presente texto toma como objeto de análisis los productos metálicos del Calcolítico y la Edad del Bronce (3050-900 cal. BC) conocidos dentro de la Submeseta Norte y la Campiña Madrileña, con especial atención a los documentados en los enterramientos en fosa. A partir de esta base documental se ha pretendido evaluar si estos objetos están vinculados con desigualdades sociales permanentes $y$, por tanto, con formas de organización política jerarquizadas, tal y como se ha planteado tradicionalmente, o si se deben atribuir a comunidades de tipo segmentario. Para tal fin se han utilizado únicamente los datos contextualizados, que han sido sometidos a diversos procedimientos estadísticos. Los resultados muestran la escasez de objetos metálicos y la falta de conexión de éstos con estructuras sociales jerarquizadas. Se propone como alternativa explicativa la permanencia de las formas de organización social de tipo segmentario durante todo el tramo temporal analizado.

\footnotetext{
ABSTRACT

This article is focused on the metal objects of the Chalcolithic and Bronze Age (3050-900 cal BC) documented in the Submeseta Norte and Campiña Madrileña, with special attention given to those found in pit graves. The aim of this paper is to assess whether these objects are linked with social inequalities, and, therefore, with

(*) Área de Arqueología. Departamento de Ciencias Históricas y Geografía. Facultad de Humanidades y Educación. Universidad de Burgos. C/ Villadiego s/n, 09001, Burgos. Correos electrónicos: ecarmona@ubu.es, arqueo@ubu.es, jmontero@ubu.es.

Recibido: 12-II-2010; aceptado: 8-V-2010.
}

hierarchical forms of political organization (as has been traditionally interpreted), or with kinship-based societies. For doing so, we have only used contextualized data, which have been analyzed with different statistical procedures. The results show a scarcity of metal objects, which, are also not connected with hierarchical social structures. Consequently, we propose, as an alternative explanation, the persistence of kinship-based societies during the analyzed period.

Palabras clave: Objetos metálicos; Enterramientos en fosa; Sociedades segmentarias; Calcolítico; Edad del Bronce; Submeseta Norte; Campiña Madrileña.

Key words: Metal objects; Pit graves; Kinship-based societies; Chalcolithic; Bronze Age; Submeseta Norte; Campiña Madrileña.

\section{INTRODUCCIÓN}

El presente trabajo forma parte de una investigación de mayor alcance que toma por objeto de discusión la caracterización de los marcos sociales de las comunidades dentro del ámbito comprendido por la Submeseta Norte y la Campiña Madrileña (Fig. 1) (1). En este marco, las etapas del Calcolítico y la Edad del Bronce (3050-900 cal. BC) constituyen unidades de análisis sobre la

(1) Dicha línea de trabajo se inserta dentro del Proyecto de Investigación Introducción al estudio interdisciplinar de las sociedades segmentarias en el centro-norte peninsular (ref. BU009A09) subvencionado por la Dirección General de Universidades e Investigación de la Consejería de Educación de la Junta de Castilla y León. 


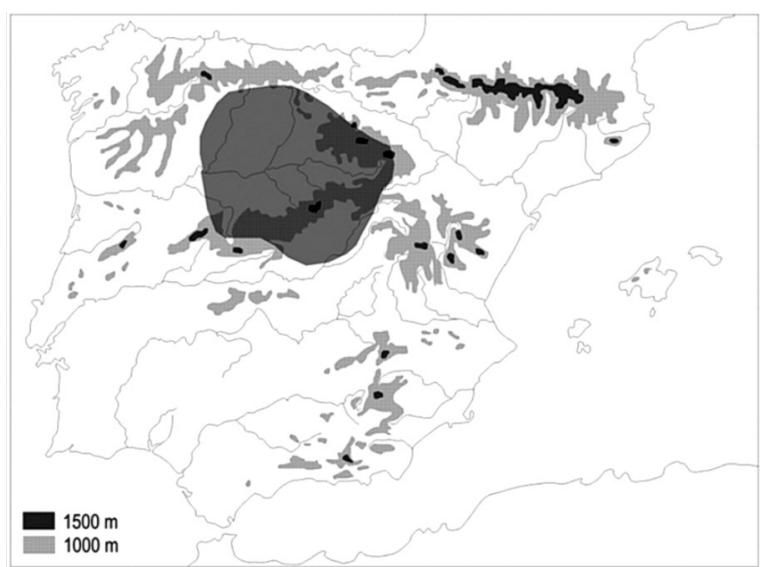

Fig. 1. Área de estudio: zona central de la Cuenca del Duero y Campiña madrileña.

Primera Edad de los Metales, donde es posible observar procesos históricos de largo recorrido, caso del mantenimiento del parentesco como relación social permanente bajo formas simples o bien jerarquizadas.

Esto último no es un tema analizado en detalle en la investigación precedente. Sí lo es, en cambio, la utilización de algunas evidencias arqueológicas como testimonio de desigualdades sociales o formas de organización complejas entendidas como "jefaturas" o "jerarquías". Este tratamiento toma como criterio principal la existencia de una "élite primigenia" cuya expresión material aparece reflejada en la acumulación de riqueza, canalizada a través de la distribución y consumo de objetos metálicos (Delibes de Castro y Fernández Manzano 2000: 101-104). De tal forma que el marco empírico lo constituyen metales procedentes de contextos arqueológicos diferenciados: lugares de hábitat, tumbas y depósitos. En base a ello, la investigación sobre la riqueza ha tomado como referencia las fluctuaciones numéricas de tales objetos registrados en las entidades arqueológicas mencionadas $\mathrm{y}$, de manera especial, en los enterramientos (Aliaga Almela 2008: 36; Bueno Ramírez et al. 2005: 67-70; Liseau et al. 2008: 117). De hecho, las "altas" cuantías de estos testimonios en determinados contextos funerarios han sido interpretadas como la prueba de la existencia de tumbas ricas y excepcionales. De este modo, la distribución diferencial de objetos metálicos ha servido para inferir la existencia de jefaturas, al menos desde los últimos momentos del Campaniforme (Delibes de Castro y Fernández-Miranda 1993; Delibes de Castro y Herrán
Martínez 2007; Delibes de Castro y Val Recio 2007-2008).

Tiene interés discutir, por tanto, si estos planteamientos resultan o no adecuados. En definitiva, el presente trabajo pretende, en primer lugar, elaborar una base documental para evaluar si el registro arqueológico es consecuente con los planteamientos explicativos histórico-culturales, donde se generan las presunciones señaladas, $y$, en segundo lugar, proponer opciones explicativas donde los datos aportados por las entidades arqueológicas respalden hipótesis formuladas desde marcos teóricos distintos, con el fin de conocer si la distribución y consumo de metales son consecuencia de un tipo de organización socioeconómica diferente a la señalada.

Con el fin de evaluar el consumo de metal, se han utilizado como punto de referencia la presencia de estos objetos en un marco general comprendido por varios contextos: hábitat, funerario y depósitos. Esta panorámica posibilita examinar cómo es el patrón general de consumo y amortización del metal dentro de las comunidades, a la vez que permite su confrontación con contextos restringidos, como las inhumaciones en fosa. La elección de estas últimas como objeto de estudio preferente se justifica por varias razones. En primer lugar, por ser el único fenómeno funerario recurrente en la Prehistoria Reciente de la Submeseta Norte y la Campiña Madrileña. En segundo lugar, por presentar menores dificultades en la asociación de individuos y objetos que les acompañan, a diferencia de los que sucede en megalitos o cuevas. En tercer lugar, porque suponen, en la mayoría de los casos, contextos arqueológicos cerrados; es decir, representa una de las pocas posibilidades de poner en relación a los individuos, inversión de trabajo y la amortización de capital, canalizada a través de los ajuares (Díaz del Río 2001: 313).

\section{BASE DOCUMENTAL Y CRITERIOS METODOLÓGICOS}

Para llevar a cabo la investigación, el primer paso ha consistido en articular una base documental representativa para poder realizar una lectura posterior en la clave anteriormente mencionada y contrastar, en consecuencia, las hipótesis planteadas. En este sentido, uno de los 
problemas con los cuales se ha topado la investigación hace referencia a la naturaleza de las fuentes, dado que éstas son muchas veces dudosas e imprecisas a la hora de referirse a la procedencia de los objetos metálicos y a los contextos donde están integrados. De hecho, el esfuerzo fundamental ha consistido en poder sistematizar una base de datos donde los objetos metálicos y sus contextos arqueológicos definidos fuesen el criterio fundamental, en detrimento de la descripción y las particularidades tipológicas de las piezas metálicas.

\subsection{Base documental}

En apariencia, la cantidad de información existente sobre la metalurgia del cobre y del bronce en las tierras del centro peninsular es abundante. Sin embargo, no todos los datos disponibles reúnen las mismas condiciones para los objetivos planteados en este trabajo.

En primer lugar, existen problemas derivados de su origen. Muchos de ellos provienen de excavaciones antiguas, con escasas referencias sobre sus contextos de procedencia como, por ejemplo, las piezas documentadas en los monumentos megalíticos de Salamanca y Zamora (Morán Bardón 1935) o en algunas cavidades de las estribaciones de la Sierra de la Demanda (Delibes de Castro 1988a: 33-113). A ello se suman aquellos otros recuperados al margen de todo control arqueológico, como sucede en la mayoría de los depósitos metálicos del Bronce Final (Fernández Manzano 1986). Tales deficiencias, añadidas a la falta de marcos teóricos ajenos al empirismo, son las que han conducido, generalmente, a plantear en los últimos años como estrategias de la investigación dos facetas preferentes: por un lado, la descripción, clasificación tipológica y ordenación temporal (Delibes de Castro y Fernández Manzano 1991; Delibes de Castro y Fernández Manzano 1986; Fernández Manzano 1986); y por otro, la faceta técnica basada en los estudios metalográficos (Blasco Bosqued y Rovira Llorens 1992-1993; Delibes de Castro y Montero Ruiz 1999; Fernández Manzano et al. 2005; Herrán Martínez 2008; Rovira Llorens 2004; Rovira Llorens y Gómez Ramos 1994), que en muchas ocasiones, cuando se utiliza en los estudios prehistóricos de la Meseta, se han orientado a suplir las deficiencias derivadas de la tipología, sin explo- rar el amplio potencial informativo que va más allá de las cuestiones técnicas, descuidando la relación que se establece entre estos aspectos respecto a los cauces de distribución, los lugares de consumo y de amortización. La aplicación de la tipología para dotar a los objetos de una atribución cultural ha dado lugar a otro problema: la ausencia de unanimidad sobre la atribución ofrecida a muchos objetos, debido al criterio subjetivo sobre el que se efectúan las atribuciones (ver por ejemplo Bellido Blanco 1994; Delibes de Castro et al. 1999; Delibes de Castro et al. 2007; Herrán Martínez 2008). A ello se suma otro problema establecido por la discriminación de las piezas metálicas con rasgos tipológicos poco expresivos, generalistas o ambiguos como, por ejemplo, los punzones y las hachas planas. El conjunto de aspectos problemáticos apuntados, no obstante, tiene una amplia repercusión en el cómputo de objetos por atribuciones o con una asignación concreta.

En segundo lugar, las atribuciones son consideradas, generalmente, etapas secuenciales. Mientras esto puede resultar adecuado para la Edad del Bronce, no ocurre lo mismo con las atribuciones culturales integradas en el Calcolítico, debido a la prolongada convivencia entre materiales pertenecientes al "Precampaniforme" y Campaniforme, por un lado, y Campaniforme y Bronce Antiguo, por otro, tal y como demuestran las dataciones absolutas disponibles que tienden a solaparse (Castro et al. 1996: 100-101; Díaz del Río 2001: 369-374; Fabián García 2006-446; Galán Sauliner 1998). Esto supone una cuestión relevante que no se explica únicamente a través de la sustitución progresiva de unas "culturas arqueológicas" por otras. Su alcance no puede ser discutido en el presente texto, dado que excede el marco del mismo. No obstante, para los fines expositivos de este artículo se ha adoptado la secuenciación progresiva de las diferentes atribuciones como herramienta adecuada para la observación de fenómenos históricos. En este sentido, se han asumido las siguientes atribuciones culturales y rangos cronológicos: Calcolítico Inicial (3050-2400 cal. BC), Calcolítico Campaniforme (2600-1900 cal. BC), Bronce Inicial (2000-1750 cal. BC), Bronce Medio (Protocogotas) (1750-1500 cal. BC), Bronce Final (Cogotas Pleno) (1500-1000 cal. BC) y Bronce Final (Depósitos del Bronce Final Atlántico) (1200-900 cal. BC). 


\subsection{Criterios metodológicos}

\subsubsection{Procedencia de los datos}

La base empírica se ha elaborado a partir de objetos metálicos procedentes de contextos precisos: lugares de hábitat, sepulturas y depósitos. Todos ellos constituyen marcos de referencia para comparar y evaluar el alcance o las pautas que adopta en cada caso la recepción de objetos metálicos. Además, tales marcos tienen otras ventajas. Por un lado, ofrecen campos discusivos a través de la amortización de tales objetos mediante las oscilaciones y cambios en la cuantificación. Por otro, su relación con las estructuras sociales supone un marco para analizar su despliegue y canalización como expresiones que originan tales cambios.

\subsubsection{Categorías de objetos}

Además de la elaboración de una base documental con entidad por sí misma, se ha tenido en cuenta una ordenación operativa de los objetos metálicos. Con el fin de mantener unos referentes comunes, se han respetado, de manera general, las categorías habituales en los estudios sobre metalurgia en la Prehistoria Reciente: armas (espadas, puntas de flecha, de lanza, regatones, puñales y alabardas), adornos (cintas, plaquitas, colgantes, torques, cuentas de collar o tocado, cápsulas, alfileres, brazaletes, anillas y fíbulas), hachas, lingotes o varillas, recipientes, utensilios (punzones, azuelas, cinceles, clavos, hojas de sierra, navajas de afeitar y yunques) y, por último, aquéllos que son indeterminados. Puede llamar la atención la distinción de las hachas como grupo independiente. Se ha optado por la separación debido a su carácter polivalente. Por un lado, tienen la condición de utensilios, cómo exponen los estudios efectuados sobre las cadenas operativas (Montero Ruiz 1992; 1993; Gener et al. 2009). No obstante, esta faceta puede matizarse dado que se ha valorado, preferentemente, la fabricación de los objetos junto a la distribución y transformación de la materia prima. Este tipo de enfoque no analiza por completo el alcance de estas piezas en los contextos de consumo y amortización. Por otro lado, otra vertiente de tales elementos aparece expuesta por su configuración como lingotes (Gómez Ramos 1993; Blasco Bosqued y Lucas Pellicer 2001: 226). Por último, sus rasgos morfológicos no descartan su utilización como elemento coercitivo, faceta que se asume de manera genérica cuando se registran en contextos específicos como los Depósitos del Bronce Final Atlántico. Las peculiaridades recogidas obligan a no descartar ninguna de ellas, de manera que quedaría justificada su consideración como categoría individualizada.

\subsubsection{Tratamiento estadístico de la información}

La información recopilada se ha transformado en versiones numéricas con las cuales se han elaborado distintas tablas y figuras. Tales datos, a su vez, han requerido una valoración específica mediante tratamientos estadísticos. Estos últimos responden a varios procedimientos del test estadístico $\chi^{2}$, los cuales se han destinado a la identificación de diferencias u homogeneidades establecidas entre los componentes de un conjunto (test de homogeneidad global) o bien, a buscar los elementos más discrepantes existentes entre dos series, donde se comparan sus respectivos efectivos (tablas dos por dos).

\subsection{Variables utilizadas}

\subsubsection{Cuantificación de los objetos metálicos: Número total de objetos conocidos}

En la actualidad el número total de ejemplares metálicos conocidos con independencia de su procedencia (con o sin contexto preciso) asciende a 1.022 piezas, los cuales aparecen repartidos de forma desigual en función de las distintas atribuciones culturales (Tab. 1). Las cuantías más notorias corresponden a los Depósitos del Bronce Final Atlántico con 278, que suponen el 27,20\%, al Calcolítico, con 272 objetos que suponen el $27,1 \%$ de dicha población; y menos a la Edad del Bronce 196, que suponen el 19,17\%. Fuera del cómputo quedan 271 sin atribución específica, que suponen el $26,51 \%$.

Sin embargo, estas cifras esconden una serie de problemas que se suman a los anteriormente comentados. De hecho, un buen número de piezas no tienen atribución definida, puesto que carecen tanto de contexto como de rasgos tipológicos específicos. Esto afecta a numerosos punzones, hachas planas y puntas de aletas y pedúnculo. Por otro lado, un alto número de piezas 


\begin{tabular}{|l|r|r|}
\hline \multicolumn{1}{|c|}{ Atribuciones } & $\begin{array}{r}\mathbf{N .}^{\mathbf{0}} \text { total } \\
\text { objetos }\end{array}$ & Porcentaje \\
\hline Calcolítico Inicial & 45 & 4,40 \\
Calcolítico Campaniforme & 232 & 22,70 \\
Bronce Inicial & 66 & 6,45 \\
Bronce Medio (PC) & 54 & 5,28 \\
Bronce Final (CP) & 76 & 7,43 \\
BF Atlántico (Depósitos) & 127 & 12,42 \\
BF Atlántico sin contexto & 137 & 13,40 \\
BF Atlántico contexto dudoso & 14 & 1,36 \\
Sin atribución & 271 & 26,51 \\
\hline Total & 1.022 & 100 \\
\hline
\end{tabular}

Tab. 1. Frecuencias absolutas y relativas según sus atribuciones.

del Bronce Atlántico también carecen de contexto $\mathrm{y}$ son atribuidas este grupo exclusivamente por sus rasgos tipológicos. Finalmente, algunas piezas del Bronce Atlántico fueron documentadas en contextos ambiguos, que presentan dudas para su adscripción cronológica y su atribución cultural.

\subsubsection{Base metálica}

Los objetos recopilados hasta este momento por la investigación tienen distintas bases metálicas: cobre, bronce, oro y plata (Tab. 2). Su reparto es desigual según los períodos, pues la mayoría de los objetos áureos se concentra en el Calcolítico Campaniforme (11 de 24) y los fabricados en plata en el Bronce Inicial (4 de 7).

\begin{tabular}{|l|r|c|}
\hline \multicolumn{1}{|c|}{ Material } & \multicolumn{1}{c|}{ N. $^{{ }^{*}}$} & Porcentaje \\
\hline Cobre & 269 & 46,62 \\
Bronce & 276 & 47,83 \\
Oro & 24 & 4,16 \\
Plata & 7 & 1,21 \\
Cobre plomado & 1 & 0,17 \\
\hline Total & 577 & 100 \\
\hline
\end{tabular}

Tab. 2. Frecuencias de objetos según su base metálica.

No obstante, las cifras expuestas tan sólo son indicativas, ya que los análisis metalográficos únicamente alcanzan a 560 objetos, lo que supone el 55,9\% de la población. Estas cifras son más escuetas (31\%) si únicamente se contabilizan los objetos que proceden de contextos definidos. En todo caso, una cuestión a subrayar es la baja cuantía de objetos fabricados en metales "nobles", pues la mayoría de los objetos están elaborados casi por igual en cobre y bronce.

\subsubsection{Peso}

Otra variable de indudable interés es la correspondiente al peso. Sin embargo, se ha omitido debido a que la información empleada procede fundamentalmente de publicaciones, en las cuales dicho dato no se suele incorporar, salvo excepciones.

\subsubsection{La entidad de la muestra utilizada}

El desarrollo de las cuestiones planteadas en el presente texto exige disponer de una información básica, como la constituida por los contextos, de la que muchas piezas carecen. La falta de este requisito, ha dado lugar a la exclusión de piezas del análisis. Así pues, la población de referencia utilizada está integrada por 471 piezas, que representan el 46,08\% respecto al conjunto total de elementos metálicos contabilizados (Tab. 1). Este número, aún tomado como muestra respecto al primero, ofrece condiciones para ser significativo; de aquí que la población estudiada se pueda, a su vez, considerar con entidad en sí misma.

Establecidos los criterios de discriminación que determinan la población operativa, es preciso comentar sus características principales. En primer lugar, se observa una distribución desigual de piezas metálicas según su atribución (Fig. 1). Tal reparto de objetos enfatiza al Calcolítico Campaniforme y a los Depósitos del Bronce Final Atlántico como dos hitos en los que la producción y consumo de objetos metálicos es superior al resto. No obstante, esta apreciación puede ser matizada en función de otros criterios.

Una forma de valorar el alcance de las cifras anteriores resulta posible a través del cálculo de la densidad de objetos por yacimientos. Este indicador propone cuantías muy bajas: no superan en ningún caso los 5 objetos por yacimiento (Fig. 2 y Tab. 3), excepto los relacionados con los Depósitos del Bronce Final Atlántico. El Calcolítico Campaniforme también queda rebajado en cuanto a importancia, aunque indica una tendencia llamativa: un incremento paulatino que, en este caso, llega hasta el Bronce Inicial. En cambio, en 


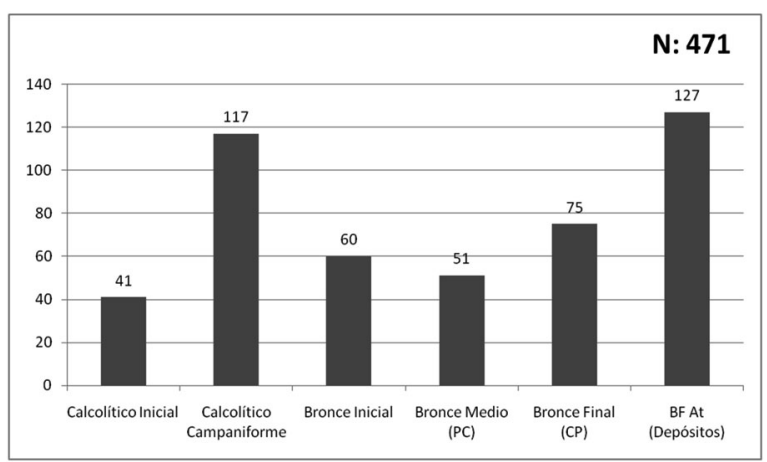

Fig. 2. Frecuencias de objetos metálicos según las atribuciones culturales.

\begin{tabular}{|c|c|c|c|}
\hline & $\begin{array}{l}\text { N. }{ }^{\circ} \text { Yaci- } \\
\text { mientos }\end{array}$ & $\begin{array}{c}\mathrm{N}^{\circ} \\
\text { Metales }\end{array}$ & Índice \\
\hline Calcolítico Inicial & 26 & 41 & 1,57 \\
\hline $\begin{array}{l}\text { Calcolítico Campa- } \\
\text { niforme }\end{array}$ & 45 & 117 & 2,62 \\
\hline Bronce Inicial & 14 & 60 & 4,28 \\
\hline Bronce Medio (PC) & 15 & 51 & 3,4 \\
\hline Bronce Final (CP) & 21 & 76 & 3,61 \\
\hline BF At. (Depósitos) & 18 & 127 & 7,05 \\
\hline
\end{tabular}

Tab. 3. Densidad de objetos por yacimiento y su relación con las distintas atribuciones culturales.

el ciclo cultural de Cogotas I a lo largo del Bronce Medio (Protocogotas) y Final (Cogotas Pleno) se observa un descenso, aunque su representación supera a lo observado durante el Calcolítico Campaniforme. La tendencia vuelve a ascender cuando se compara con los Depósitos del Bronce Final Atlántico. En definitiva, se aprecia un incremento paulatino en el consumo del metal en el tramo histórico analizado, desde los primeros momentos (Calcolítico Inicial) a los finales (Depósitos del Bronce Final Atlántico).

Otro aspecto de interés aparece constituido por las cuantías y representación que muestran las categorías de objetos establecidas. Según la representación se distinguen tipos dominantes y otros secundarios (Fig. 3). Entre los primeros se reconocen armas y utensilios, mientras que los segundos aparecen constituidos por hachas, adornos y lingotes.

Una primera lectura revela la importancia que adquieren las armas junto a los utensilios en el conjunto. A partir de estos datos, interesa

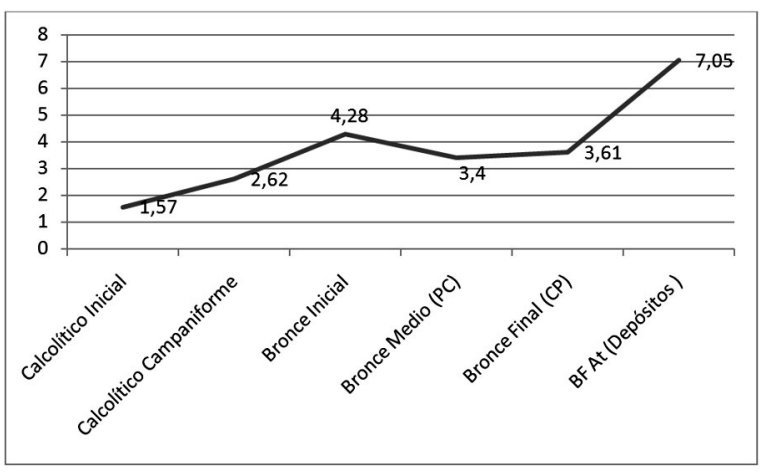

Fig. 3. Representación gráfica elaborada a partir de los datos contenidos en la tabla 3.

comprobar si entre las distintas categorías existe algún tipo de relación. Con esta finalidad se ha realizado la figura 4, que muestra los distintos grupos de objetos en función de las atribuciones.

La asociación de las frecuencias señala la ausencia de una acumulación progresiva de un determinado tipo de objeto. Por el contrario, muestra dos tipos más proclives a acumular un mayor número en la representación: las armas y los utensilios. Sin embargo, estas frecuencias más altas corresponden a momentos cronológicos distintos. Las armas son mayoritarias en el Calcolítico Campaniforme $(63,72 \%)$ y Bronce Final: Cogotas Pleno $(32,91 \%)$ y Depósitos del Bronce Final Atlántico (49,15\%), mientras que los utensilios lo son en el Calcolítico Inicial (55\%), Bronce Inicial (58,73\%) y Bronce Medio (Protocogotas) $(48 \%)$.

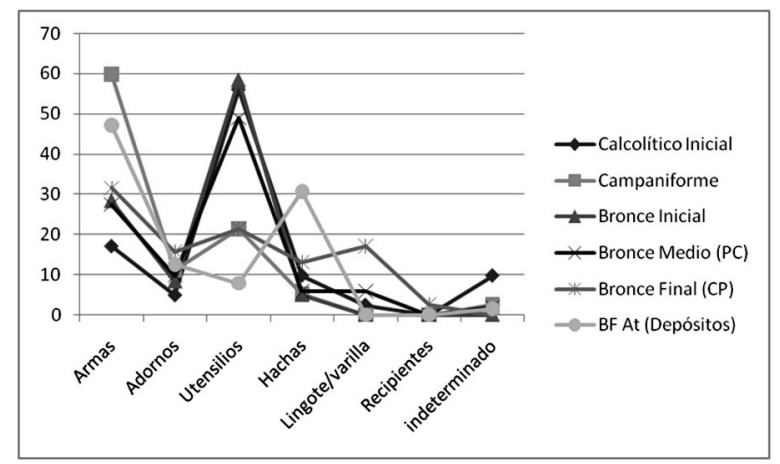

Fig. 4. Porcentajes atendiendo a su correspondencia con las distintas categorías de objetos y atribuciones culturales. 


\section{METALES Y CONTEXTOS}

Como se ha indicado, la población de piezas metálicas utilizada en el trabajo está integrada por ejemplares procedentes de distintos contextos (Tab. 4). De todos ellos -lugares de hábitat, depósitos y sepulturas-, los que más aportan son los primeros. Éstos, no obstante, definen ámbitos de escasa personalidad, poco significativos, ya que no proceden de viviendas, ni tampoco de lugares de actividades domésticas o productivas, sino de estratigrafías imprecisas (Fabián García 2006; Jimeno Martínez 1984; Val Recio 1992) e, incluso, caracterizados como productos de desecho rellenando estructuras excavadas en el suelo con morfología de hoyos, habitualmente silos que han perdido sus funciones originales (Blasco Bosqued et al. 2004; Celis Sánchez et al. 2007).

Frente a lo que sucede en los lugares de hábitat, las cuantías más bajas de productos metálicos aparecen asociadas a contextos específicos, como enterramientos. No obstante, una excepción a esta orientación aparece representada por el Calcolítico Campaniforme.

Junto a la anterior, es necesario añadir una manifestación arqueológica muy concreta, definida por los depósitos correspondientes al Bronce Final avanzado, entre el 1200 y el 900 cal. BC; o lo que es lo mismo, vinculada a cronologías que, según la periodización europea, se prolongan hasta el Bronce Final II y IIIa (Delibes de Castro y Fernández Manzano 1991: 206 y 208; Delibes de Castro et al. 2001: 81; Fernández-Posse y Montero Ruiz 1998: 195).

La descripción de los lugares de procedencia plantea otras cuestiones; ¿qué relación guardan los tipos de objetos con los marcos de amortización? ¿Cuál es el alcance de la relación establecida? Con el fin de responder a las cuestiones planteadas se han sometido los datos recogidos en la tabla 4 a varios tratamientos estadísticos. El proceso presenta dos vertientes. Por un lado, se ha analizado la homogeneidad global del conjunto mediante el test estadístico de $\chi^{2}$. Por otro, se han buscado los grupos más anómalos causantes de las diferencias sensibles a la evaluación estadística. En este caso, se ha utilizado la comparación entre dos series, a través del test $\chi^{2}$ (tablas $2 \times 2$ ).

La evaluación de los tipos de objetos respecto a los lugares de amortización (tumbas y hábitat) ofrece resultados que señalan la ausencia de una distribución homogénea $\left(\mathrm{gl}=4 ; \chi^{2}=53,16\right.$; $\mathrm{p}=>0,0001)$. El complemento del análisis precedente, efectuado comparando las series que determinan las tumbas y los lugares de hábitat, mediante comparación el test $\chi^{2}$ (tablas $2 \times 2$ ), indica unas diferencias altamente significativas de las armas y los utensilios, las primeras en favor de las tumbas y los segundos de los hábitats (Tab. 5). Asimismo, se identifican diferencias significativas en el grupo de hachas y adornos, las primeras a favor de los hábitat, mientras que el de los adornos tiene mayor presencia en las tumbas. Frente a estas diferencias, el único grupo homogéneo resulta ser el correspondiente a los lingotes-varillas. Un rasgo particular que ofrecen los datos estadísticos consiste en la estrecha relación entre armas y adornos en contextos funerarios. La vinculación entre ambos tipos de objetos parece tener mayor consistencia durante el Calcolítico Campaniforme. En los demás casos, aunque las frecuencias bajas impiden ver detalles, no se establece una estrecha relación entre ambos tipos de objetos.

Junto al análisis anterior, se han realizado otros paralelos donde se evalúan los contextos de amortización de los metales respecto a las atribuciones culturales. La valoración estadística del conjunto muestra una distribución no homogénea $\left(\mathrm{gl}=4 ; \chi^{2}=187,24 ; \mathrm{p}=>0,0001\right)$. El análisis posterior comparando las series delimitadas por los lugares de amortización (tumbas y hábitat) mediante el test $\chi^{2}$ (tabla $2 \times 2$ ) señala diferencias significativas en todos los momentos respecto a los contextos de amortización de los objetos metálicos (Tab. 7). Estos resultados también ponen de manifiesto que durante el Calcolítico Campaniforme la representación principal de los objetos metálicos se vincula a las sepulturas, situación inversa a la que exponen los restantes períodos (Tab. 7).

Otro de los factores que se ha evaluado hace referencia a la circulación y uso restringido de los objetos metálicos. Para examinar tales cuestiones se han elaborado dos marcos de representación; por una parte la tabla 6 , donde se recogen los elementos coercitivos y de ostentación, según su distribución por períodos $\mathrm{y}$, por otra, la figura 6 , que muestra los objetos anteriores comparados con los bienes utilitarios (utensilios, hachas y lingotes). Estos datos se han sometido también a un doble tratamiento estadístico. La valoración del conjunto enfatiza una diferencia altamente significativa $\left(\mathrm{gl}=5 ; \chi^{2}=53,37 ; \mathrm{p}=>0,0001\right)$. El se- 


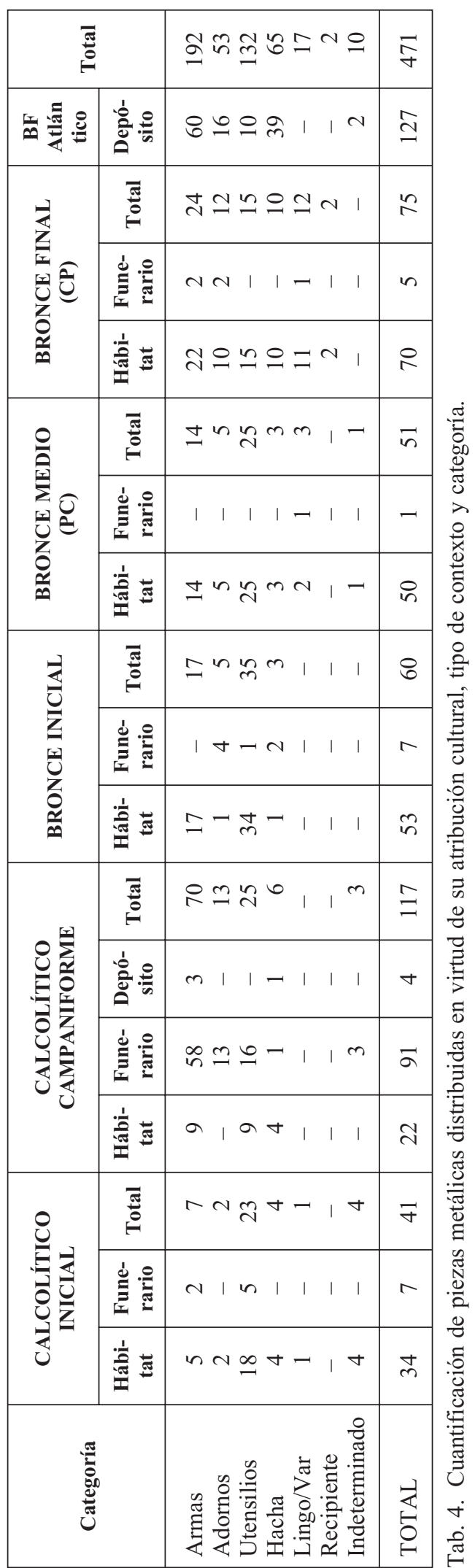

T. P., 67, N. ${ }^{\circ} 2$, julio-diciembre 2010, pp. 373-387, ISSN: 0082-5638 doi: $10.3989 /$ tp. 2010.10045 


\begin{tabular}{|l|c|c|c|c|}
\hline & Tumbas & Hábitat & $\chi^{\mathbf{2}(2 \times \mathbf{2})}$ & Diferencia \\
\hline Armas & $62(57,4 \%)$ & $67(30,2 \%)$ & 22,621 & A.S. \\
Utensilios & $22(20,4 \%)$ & $101(45,5 \%)$ & 19,617 & A.S. \\
Hachas & $3(2,8 \%)$ & $22(9,9 \%)$ & 5,278 & S. \\
Adornos & $19(17,6 \%)$ & $18(8,1 \%)$ & 6,565 & S. \\
Lingotes/varillas & $2(1,9 \%)$ & $14(6,3 \%)$ & 3,125 & H. \\
\hline Total & 108 & 222 & & \\
\hline
\end{tabular}

Tab. 5. Resultados obtenidos a través de la comparación $\chi^{2}$ (A.S. = altamente significativa, S. = significativa; H. = Homogéneo).

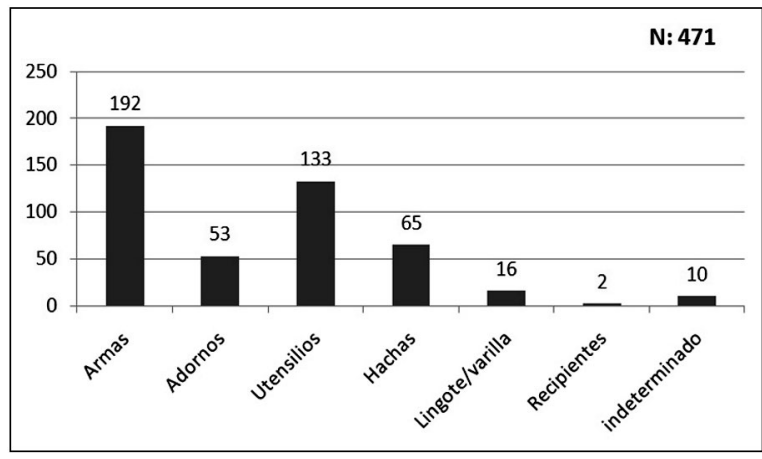

Fig. 5. Cuantías de piezas metálicas según las categorías de objetos establecidas.

\begin{tabular}{|l|c|c|}
\hline & \multicolumn{1}{|c|}{ Armas } & \multicolumn{1}{c|}{ Adornos } \\
\hline Calcolítico Inicial & $2(3,22 \%)$ & $0(0 \%)$ \\
Calcolítico Campaniforme & $58(93,54 \%)$ & $13(68,42 \%)$ \\
Bronce Inicial & $0(0 \%)$ & $4(21,05 \%)$ \\
Bronce Medio (PC) & $0(0 \%)$ & $0(\%)$ \\
Bronce Final (CP) & $2(3,22 \%)$ & $2(10,52 \%)$ \\
\hline Total & 62 & 19 \\
\hline
\end{tabular}

Tab. 6. Diferencias entre las armas y adornos según las atribuciones culturales.

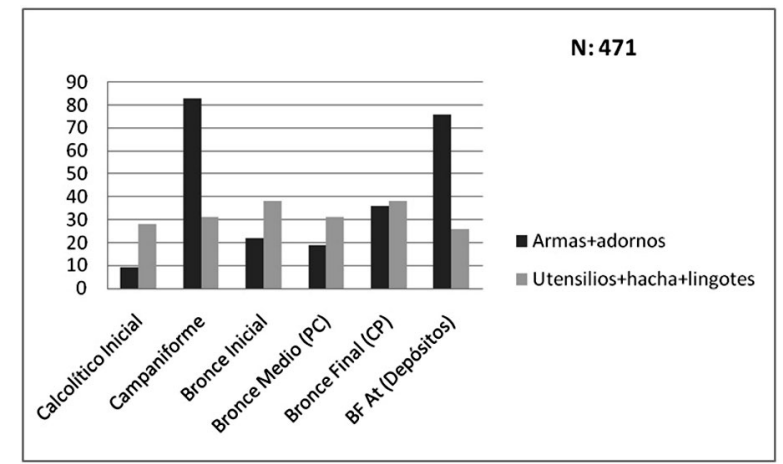

Fig. 6. Cuantificación de piezas agrupadas según su consideración como elementos coercitivos-suntuarios (armas y adornos) y bienes utilitarios (utensilios, hachas y lingotes).

gundo (Tab. 8) expone los resultados de la comparación de las series (armas y adornos, respecto a hachas, utensilios y lingotes) mediante el test estadístico $\chi^{2}$ (tabla $2 \times 2$ ). Durante el Calcolítico Campaniforme y los Depósitos del Bronce Final Atlántico las frecuencias más notorias corresponden a las armas y adornos, con diferencias, en un caso, altamente significativas y, en otro, significativas. Por el contrario, en el Calcolítico Inicial, en el Bronce Inicial y Bronce Medio (Protocogo-

\begin{tabular}{|l|c|c|r|c|}
\hline & Tumbas & Hábitat & $\chi^{\mathbf{2}(2 \times \mathbf{2})}$ & Diferencia \\
\hline Calcolítico Inicial & $7(6,3 \%)$ & $34(14,8 \%)$ & 5,143 & S. \\
Calcolítico Campaniforme & $91(82 \%)$ & $22(9,6 \%)$ & 176,486 & A.S. \\
Bronce Inicial & $7(6,3 \%)$ & $53(23,1 \%)$ & 14,585 & A.S. \\
Bronce Medio (PC) & $1(0,9 \%)$ & $50(21,8 \%)$ & 25,694 & A.S. \\
Bronce Final (CP) & $5(4,5 \%)$ & $70(30,6 \%)$ & 29,538 & A.S. \\
\hline Total & 111 & 229 & & \\
\hline
\end{tabular}

Tab. 7. Resultados obtenidos a través de la comparación $\chi^{2}$ (A.S. = altamente significativa, S. = significativa; H. = Homogéneo). 


\begin{tabular}{|l|c|c|c|c|}
\hline & Armas + adornos & Hachas + utensilios + lingotes & $\chi^{\mathbf{2}} \mathbf{( 2 \times \mathbf { 2 } )}$ & Diferencia \\
\hline Calcolítico Inicial & $9(3,7 \%)$ & $28(13,1 \%)$ & 13,650 & A.S. \\
Calcolítico Campaniforme & $83(33,9 \%)$ & $31(14,5 \%)$ & 23,009 & A.S. \\
Bronce Inicial & $22(9 \%)$ & $38(17,8 \%)$ & 7,745 & S. \\
Bronce Medio (PC) & $19(7,8 \%)$ & $31(14,5 \%)$ & 5,331 & S. \\
Bronce Final (CP) & $36(14,7 \%)$ & $37(17,3 \%)$ & 0,575 & H. \\
BF At. (Depósitos) & $76(31 \%)$ & $49(22,9 \%)$ & 3,804 & S. \\
\hline Total & 258 & 214 & & \\
\hline
\end{tabular}

Tab. 8. Resultados obtenidos a través de la comparación $\chi^{2}$ (A.S. = altamente significativa, $\mathrm{S}$. = significativa; H. = Homogéneo).

tas) la mayor representación está a favor de las hachas, utensilios y lingotes. Sólo la representación correspondiente a la etapa del Bronce Final (Cogotas Pleno) es homogénea. En conclusión, el consumo diferencial de objetos coercitivos y de adorno personal sólo se identifica en los períodos correspondientes al Calcolítico Campaniforme y Bronce Final Atlántico relacionado con los Depósitos.

\section{OBJETOS METÁLICOS Y ENTERRAMIENTOS EN FOSA}

Tradicionalmente se ha venido aceptando que la materialización de la riqueza en la Prehistoria Reciente está asociada a la presencia de metales sobre todo en dos tipos de contextos: funerarios y depósitos. De tal forma que la existencia de una comunidad con diferencias sociales presupone un consumo y deposición diferencial de este tipo de objetos. Los datos anteriormente expuestos constituyen una base empírica para debatir esta cuestión.

El conocimiento disponible sobre el ritual funerario de la Prehistoria Reciente en el escenario objeto de estudio, la Submeseta Norte y la Campiña Madrileña, pone de relieve un panorama poco uniforme, expresado mediante la utilización de diferentes ámbitos, con independencia de los períodos, para depositar a los difuntos: megalitos, cuevas y fosas (Delibes de Castro y Santonja 1986; Blasco 1997; Fabián 1995; Esparza Arroyo 1990; Garrido Pena 2000: 49-58; Rojo Guerra et al. 2005; Esparza Arroyo et al. 2008; Aliaga Almela 2008).

A pesar de la diversidad de escenarios y fórmulas funerarias, lo cierto es que se reconoce una faceta compartida representada por el ritual constituido por la inhumación. En las fosas, éste aparece establecido por la disposición de los cuerpos, ya sea de forma individual o múltiple, en el fondo de las mismas. Además, otra faceta particular lo constituye la recurrente presencia de elementos depositados dentro de tales sepulturas. En la mayoría de los casos estos elementos se suelen interpretar como ajuares u ofrendas, siendo dentro de la primera categoría donde se incluyen los objetos metálicos (Aliaga Almela 2008; Díaz del Río 2001: 149; Garrido Pena 2000: 61-66; Macarro 2002: 120; Vázquez Cuesta 2009).

La cuestión, entonces, consiste en dirimir si, los objetos metálicos asociados a inhumaciones en contenedores con morfología de fosa son el reflejo directo de disimetrías sociales, como mantienen las corrientes empiristas (Delibes de Castro y Val Recio 2007-2008; Delibes de Castro et al. 1995: 57; Esparza Arroyo 1990: 134; Liseau et al. 2008). Este propósito, no obstante, presenta algunas limitaciones causadas por dos aspectos: el bajo número de enterramientos y la ausencia de necrópolis segregadas de los lugares de hábitat o bien en el subsuelo de los mismos. Los pocos casos conocidos de aquellas manifestaciones ofrecen una cuantía de difuntos por yacimiento muy escasa, a lo que se añade una información reducida sobre la edad y el sexo de los individuos. Todo ello supone un obstáculo para establecer asociaciones relevantes entre los elementos que configuran los contextos funerarios.

Los datos expuestos en la tabla 9 aluden a todas las entidades de la naturaleza apuntada disponibles en la actualidad. A partir de dicha información se obtienen dos conclusiones fundamentales: el escaso número de evidencias metálicas y el exiguo contenido de ajuares. Especialmente la segunda observación sugiere un patrón de consumo 


\begin{tabular}{|c|c|c|c|c|}
\hline & N. ${ }^{\circ}$ tumbas & $\mathrm{N} .{ }^{\circ}$ fosas & N. ${ }^{\circ}$ fosas con metal & N. ${ }^{\circ}$ metales \\
\hline Calcolítico Inicial & 35 & 26 & 2 & 7 \\
\hline Calcolítico Campaniforme & 72 & 33 & 17 & 45 \\
\hline Bronce Inicial & 6 & 4 & 1 & 3 \\
\hline Bronce Medio (PC) & 17 & 17 & 1 & 1 \\
\hline Bronce Final (CP) & 6 & 3 & 2 & 5 \\
\hline Total & 136 & 83 & 22 & 65 \\
\hline
\end{tabular}

Tab. 9. Frecuencias de inhumaciones en fosa por atribuciones, fosas con elementos metálicos y cuantía total de objetos presentes en estas últimas.

escaso respecto a los objetos metálicos. O lo que es lo mismo, los datos proponen una inversión reducida en todo lo referente a este tipo de sepulturas: elaboración y gastos en ajuares, cuya única excepción aparece vinculada al Campaniforme.

\section{CARACTERÍSTICAS DE LA INFORMACIÓN APORTADAS POR LOS METALES VINCULADOS A CONTEXTOS (3300-900 CAL BC)}

La revisión efectuada, tomando como referencia los datos procedentes de contextos definidos, y el proceso de valoración desplegado permiten establecer una serie de aspectos que difieren de los presupuestos mantenidos por la vía explicativa histórico-cultural. Tal diferencia se resume en los puntos señalados a continuación:

1. Una constante, con excepción del Campaniforme y los Depósitos del Bronce Final Atlántico, definida por la pequeña escala de producción del metal y, a su vez, de acumulación del mismo.

2. Representación mayoritaria de los objetos en los lugares de hábitat, exceptuando el Campaniforme y los Depósitos del Bronce Final Atlántico.

3. Predominio de los utensilios. La producción de metal se canaliza fundamentalmente hacia la configuración de objetos funcionales, especialmente durante la Edad del Bronce.

4. La acumulación de objetos metálicos en contextos funerarios es escasa. Las cifras de los enterramientos campaniformes, dentro de este marco, suponen una excepción.

5. Presencia baja de medios de coerción (armas) a lo largo del tramo estudiado, cuyas únicas discrepancias las incorporan el Calcolítico Cam- paniforme y los Depósitos del Bronce Final Atlántico.

6. Presencia baja de objetos suntuarios (adornos y, especialmente, los elaborados con metales nobles), sin oscilaciones importantes a lo largo del ciclo estudiado, es decir, manifestaciones sin correlación con lo esperado en formas de organización social jerarquizadas, donde los adornos, al igual que los elementos coercitivos, suponen expresiones de poder.

\section{DISCUSIÓN}

La investigación centrada en la Prehistoria Reciente dentro del ámbito geográfico que sirve de marco al presente estudio ha orientado sus esfuerzos hacia la clasificación de los objetos y a la determinación de su adscripción temporal. Este enfoque ha dejado al margen otras vertientes de estudio, fundamentalmente, las que tienden a relacionar los datos con una estructura social coherente y contrastable con las peculiaridades arqueológicas.

En este sentido los intentos más próximos han estado presididos por la extrapolación de modelos de autores anglosajones propuestos para otros espacios europeos (véase, por ejemplo, Clarke 1976; Hayden 1995; Sherrat 1981, 1987). Por otra parte, las evidencias arqueológicas donde aparece un consumo de objetos de valor destacado, caso de las tumbas del Calcolítico Inicial como el Ollar de Donhierro (Segovia) (Delibes de Castro 1988b) y, en especial, las campaniformes, como Perro Alto en Fuente Olmedo (Va1ladolid) (Delibes de Castro y Herrán Martínez 2007: 189-193; Martín Valls y Delibes de Castro 1989: 11-28) o los hipogeos de Camino de las Yeseras en San Fernando de Henares (Madrid) (Li- 
seau et al. 2008), se han utilizado para sustentar la idea de progreso lineal sobre el desarrollo de la organización social, aceptando, a partir de estos puntos de referencia, diferencias sociales progresivas con sentido diacrónico. Ahora bien, dicho proceso no sólo carece de una adecuada corroboración, sino que tampoco justifica la complejidad creciente implícita en la propuesta.

El sentido lineal propuesto queda en evidencia ante unas manifestaciones arqueológicas que revelan períodos donde el consumo de metal tiene notoriedad y aparece vinculado a lugares diferenciales, al que siguen otros caracterizados por lo contrario. Ejemplos en este último sentido se reconocen en las tumbas en fosa asociadas al ciclo de la Edad del Bronce. En sentido contrario se manifiestan (al menos el número de objetos metálicos es más significativo) las tumbas del Calcolítico Campaniforme y Depósitos del Bronce Final Atlántico.

Desde otras posturas interpretativas se puede explicar la oscilación en el consumo de metal que muestran las evidencias arqueológicas como consecuencia del proceso "acumulación primitiva", a través de la cual se vincula a los productores (los primeros campesinos) con los medios de producción (fundamentalmente la tierra) (Vicent García 1995: 178). Para que la acumulación primitiva sea efectiva son necesarias unas condiciones previas: medios de apropiación permanente de la tierra, la creación de un fondo de trabajo en estrecha relación con el "capital agrario" (tierra e inversiones para ponerla en producción) y la emergencia del parentesco genealógico. Este último, al distinguir a los componentes de la comunidad mediante diferencias de rango y estatus, posibilita el acceso y la distribución desigual de los excedentes dentro de las mismas. Un escenario, en definitiva, donde la acumulación primitiva y su consolidación se fundamentan en contradicciones desplegadas entre productores y no productores.

La perspectiva de larga duración contemplada en este estudio permite poner a prueba teoría y datos, en este caso los asociados a los objetos metálicos. En este sentido, se postulan fases acumulativas para el Calcolítico Inicial y Calcolítico Campaniforme, a la que parece suceder un desplome, que comprende la Edad del Bronce, y un nuevo repunte del consumo de objetos metálicos (sobre todo coercitivos), asociado a los Depósitos del Bronce Final Atlántico.

La evidencia empírica del ciclo acumulativo entre el Calcolítico Inicial y el Calcolítico Cam- paniforme aparece bien relatada por la diferencia del tipo de objetos (Tab. 8) y los lugares de amortización diferenciales, que en el Calcolítico Campaniforme aparece expuesto mediante las tumbas (Tab. 7). En cambio, durante la Edad del Bronce, fundamentalmente tienen notoriedad los objetos utilitarios (Tab. 8), que, en consecuencia, se encuentran documentados en su mayor parte dentro de lugares de hábitat (Tab. 7). La ruptura respecto a esta última etapa que protagonizan los Depósitos del Bronce Final Atlántico aparece definida por un ascenso en el consumo de metal (Figs. 1 y 2) orientado hacia unos determinados tipos de objetos, como se constata en las altas frecuencias que adoptan tanto las armas como las hachas (Tab. 4).

\section{CONCLUSIONES}

Las entidades tomadas como elementos de análisis preferentes, los enterramientos en fosa, suponen un marco favorable para el examen planteado en estas páginas, dado que representan un sistema funerario que, a partir de condición transversal, permite -mejor que otras entidades arqueológicas- disponer de indicadores para observar y evaluar el alcance que tiene la acumulación diferencial de objetos metálicos. Ambos aspectos -el sistema de enterramiento y ajuares- han sido recogidos por la explicación tradicional para documentar diferencias sociales, recogidas bajo términos tan dispares como "régulos", "prínceps" o "aristocracia" (Delibes de Castro y Fernández-Miranda, 1993, Delibes de Castro y Val Recio, 2007-2008, Delibes de Castro y Herrán Martínez, 2007, Delibes de Castro, Romero Carnicero y Abarquero Moras, 2000). Un marco propicio para esta cuestión lo representa el Calcolítico Campaniforme, a tenor de su registro funerario y los ajuares metálicos entendidos como elementos de valor. Todo ello ha dado pie a explicar tal registro como exponente de una diferenciación social estructurada bajo esquemas de jefatura. En definitiva, si la observación es correcta, la extensión de este concepto en el tiempo (del Calcolítico Campaniforme en adelante) supondría un cambio en el Modo de Producción, que partiendo de las jefaturas culminaría en las sociedades estratificadas, y cuyos efectos se tendrían que ver reflejados con cambios sustanciales en la estruc- 
tura social. Tales cambios, no son explícitos a través de los componentes arqueológicos.

Sin embargo, frente a tal explicación, la información arqueológica disponible en la actualidad en el centro de la Península Ibérica encaja mejor con hipótesis generadas desde el materialismo histórico. Desde esta óptica, la Prehistoria Reciente se integra en un mismo Modo de Producción, correspondiente a su vez a un mismo orden social. Ahora bien, este orden social tiene dos condiciones básicas: un margen para la explotación y la resistencia de las comunidades ante esto último (Vicent García 1995: 178).

La primera condición permite una explotación que no conduce a formas permanentes de apropiación (Díaz del Río 1995: 105-106; Vicent García 1990: 274; 1995), posibilitando, de este modo, un consumo diferencial de bienes, en particular aquéllos con valor añadido como armas y objetos metálicos suntuarios. Asimismo, está detrás de ciertas manifestaciones arqueológicas vinculadas con la desigualdad, caso de las tumbas ricas del Campaniforme $\mathrm{y}$, también, con otras evidencias de carácter coercitivo (murallas, fosos, etc.). La segunda condición hace referencia al hecho de que las desigualdades no lleguen a perpetuarse; o lo que es lo mismo, no se creen formas permanentes de apropiación.

Todo este marco teórico se puede ver reflejado en el registro arqueológico del ciclo histórico estudiado, sobre todo en las tumbas de fosa. Estas entidades arqueológicas son escasas y poco elaboradas. El metal apenas está integrado en los ajuares. Los objetos de este tipo se corresponden preferentemente con bienes utilitarios. Sin embargo, no se correlacionan con la producción agrícola primaria, dado que en su mayoría son punzones y pocas hachas. La ausencia de manifestaciones que aludan a diferencias sociales permanentes remitiría a organizaciones sociales que se mantienen estructuradas dentro de las formas segmentarias.

\section{AGRADECIMIENTOS}

Con este trabajo se ha querido contribuir al homenaje al Dr. Salvador Rovira Llorens celebrado en Madrid en noviembre de 2009 en el marco del congreso Archaeometallurgy: Technological, Economic and Social Perspectives in Late Prehistoric Europe. También queremos dar las gracias al Dr. Fernando Lara Ortega de la Universidad de
Burgos por la ayuda prestada en la realización de los análisis estadísticos.

\section{BIBLIOGRAFÍA}

Aliaga Almela, R. 2008: "El mundo funerario calcolítico de la Región de Madrid". Cuadernos de Prehistoria y Arqueología de la Universidad Autónoma de Madrid 34: 23-39.

Bellido Blanco, A. 1994: "En torno a la cuestión de la metalurgia de Cogotas I: un análisis contextual". Espacio, Tiempo y Forma. Prehistoria y Arqueología 7: 191-210.

Blasco Bosqued, M.C. 1997: "Manifestaciones funerarias de la Edad del Bronce en la Meseta". Saguntum 30: 173-190.

Blasco Bosqued, M.C. y Lucas Pellicer, M.R. 2001: "Problemática del Bronce Final en la Meseta". Spal 10: 221-233.

Blasco Bosqued, M.C. y Rovira Llorens, S. 19921993: "La metalurgia del cobre y del bronce en la región de Madrid". Tabona: Revista de prehistoria $y$ de arqueología 8 (2): 397-415.

Blasco Bosqued, M.C.; Calle Pardo, J. y Sánchez-Capilla Arroyo, M.L. 2004: "La metalurgia del Bronce Medio y final (Protocogotas y Cogotas I): evolución y contexto". Zona Arqueológica 4. Miscelánea en homenaje a Emiliano Aguirre. Volumen IV. Arqueología: 49-65.

Bueno Ramírez, P.; Barroso Bermejo, R. y Balbín Berhmann, R. 2005: "Ritual Campaniforme, ritual colectivo: La necrópolis de cuevas artificiales del Valle de las Higueras, Huecas, Toledo". Trabajos de Prehistoria 67 (2): 67-90.

Castro, P.; Lull, V. y Micó, R. 1996: “Cronología de la Prehistoria Reciente de la Península Ibérica y Baleares (c. 2800-900 cal ANE)". BAR International Series 652.

Celis Sánchez, J.; Delibes de Castro, G.; Fernández Manzano, J. y Grau Lobo, L. 2007: El hallazgo leonés de Valdevimbre y los depósitos del Bronce Final atlántico en la Península Ibérica. Colección Estudios y Catálogos, 17. Junta de Castilla y León. Valladolid.

Clarke, D. 1976: "The Beaker network-social and economic models". En J.N. Lanting y J.D. Van der Waals, (eds.): Glockenbecher Symposium (Oberried, 1974): 459-77.

Delibes de Castro, G. 1988a: "La Edad del Bronce". En G. Delibes de Castro, A. Esparza Arroyo, E. García Soto, J.R. López Rodríguez y M. Mariné Isidro (eds.): La colección arqueológica del padre Saturio González en Santo Domingo de Silos. Diputación Provincial de Burgos. Burgos: 33-113. 
- 1988b: "El enterramiento calcolítico en fosa de "El Ollar", Donhierro (Segovia)". Espacio, Tiempo y Forma. Prehistoria I: 227-38.

Delibes de Castro, G. y Fernández Manzano, J. 1986: "Metalurgia del Bronce Final en la Meseta Norte: Nuevos datos para su estudio". Boletín del Seminario de Estudios de arte y Arqueología LII: 5-23.

- 1991: "Relaciones entre Cogotas I y el Bronce Final Atlántico en la Meseta Norte Española". En Actes du Premier Colloque du Parc Archéologique de Beynac. Perigueux: 203-12.

- 2000: "La trayectoria cultural de la Prehistoria Reciente (6400-2500 BP) en la Submeseta Norte española: principales hitos de un proceso". En Pré-historia recente da Península Ibérica, Actas do $3 .{ }^{\circ}$ Congresso de Arqueologia Peninsular IV, Vila Real, 1996, Porto: 95-122.

Delibes de Castro, G. y Fernández-Miranda, M. 1993: Los orígenes de la civilización. El Calcolítico en el Viejo Mundo. Historia Universal, 5 Prehistoria. Síntesis. Madrid.

Delibes de Castro, G. y Herrán Martínez, J.I. 2007: La Prehistoria. Diputación de Valladolid. Valladolid.

Delibes de Castro, G. y Santonja, M. 1986: El fenómeno megalítico en la provincia de Salamanca. Salamanca: Diputación de Salamanca.

Delibes de Castro, G. y Val Recio, J. 2007-2008: “La explotación de la sal el término de la Edad del Cobre en la Meseta Central española: ¿Fuente de riqueza e instrumento de poder de los jefes de Ciempozuelos?". Veleia. 24-25: 791-811.

Delibes de Castro, G.; Fernández Manzano, J. y Herrán Martínez, J.I. 1999: "Submeseta Norte". En G. Delibes de Castro y I. Montero Ruiz (eds.): Las primeras etapas metalúrgicas en la Península Ibérica. II. Estudios Regionales. Madrid: 63-94.

Delibes de Castro, G.; Fernández Manzano, J. y Herrán Martínez, J.I. 2007: "Los bronces de Valdevimbre y la metalurgia de Cogotas I". En J. Celis Sánchez, G. Delibes de Castro, J. Fernández Manzano y L. Grau Lobo (eds.): El hallazgo leonés de Valdevimbre y los depósitos del Bronce Final Atlántico en la Península Ibérica. Colección Estudios y Catálogos, 17. Diputación de León y Junta de Castilla y León. León: 106-31.

Delibes de Castro, G.; Romero Carnicero, F. y Abarquero Moras, F.J. 2000: "Cerámicas excisas de discutible filiación Cogotas I en el Bronce Tardío de la Península Ibérica: una taza de estilo Duffaits procedente de la Cueva del Asno (Los Rábanos, Soria)". Soria Arqueológica 2: 97-130.

Delibes de Castro, G.; Romero, F.; Sanz, C., Escudero, Z. y San Miguel, L. 1995: "Panorama arqueológico de la Edad del Hierro en el Duero Medio". En F. Romero y A. Morales (eds.): Arqueología y Medio Ambiente. El Primer Milenio a.C. en el Duero Medio. Valladolid: 49-156.
Delibes de Castro, G.; Fernández Manzano, J.; Romero Carnicero, F.; Herrán Martínez, J.I. y Ramírez Ramírez, M.L. 2001: "Metal production at the End of Late Bronze Age in the Central Iberian Peninsula". Journal of Iberian Archaeology. Adecap 3: 73-95.

Delibes de Castro, G. y Montero Ruiz, I. (eds.) 1999: Las Primeras Etapas Metalúrgicas en la Península Ibérica. II. Estudios Regionales. Instituto Universitario y Fundación Ortega y Gasset. Madrid.

Díaz del Río, P. 1995: “Campesinado y gestión pluriactiva del ecosistema: un marco teórico para el análisis del III y el II milenios a.C. en la Meseta peninsular". Trabajos de Prehistoria 52 (2): 99-109.

Díaz del Río, P. 2001: La formación del paisaje agrario: Madrid en el III y II milenios BC. Arqueología, Paleontología y Etnografía. Consejería de las Artes de la Comunidad de Madrid. Madrid.

Esparza Arroyo, A. 1990: "Sobre el ritual funerario de Cogotas I". Boletín de Estudios del Seminario de Arte y Arqueología LVI: 106-43.

Esparza Arroyo, A.; Delibes de Castro, G.; Velasco Vázquez, J. y Cruz Sánchez, P.J. 2008: "Historia de un golpe en la cabeza: Sobre el enterramiento calcolítico del Hoyo 197 de 'El Soto de Tovilla' (Tudela de Duero, Valladolid)". Boletín del Seminario de Estudios de Arte y Arqueología LXXIV: 9-48.

Fabián García, F.J. 1995: El aspecto funerario durante el Calcolítico e inicios de la Edad del Bronce en la Meseta Norte. Universidad de Salamanca, Salamanca.

Fabián García, F.J. 2006: El IV y III milenio a.C. en el Valle del Amblés (Ávila). Arqueología en Castilla y León. Monografías, 5. Junta de Castilla y León. Valladolid.

Fernández-Posse, M.D. y Montero Ruiz, I. 1998: “Una visión de la metalurgia atlántica en el interior de la Península Ibérica". En S. Oliveira Jorge (ed.): Existe una Idade do Bronze Atlântico? Trabalhos de Arqueología 10. Lisboa: 192-202.

Fernández Manzano, J. 1986: Bronce Final en la Meseta Norte española: el utillaje metálico. Colección Monografías Arqueológicas de la Junta de Castilla y León. Almazán.

Fernández Manzano, J.; Herrán Martínez, J.I. y Rovira Llorens, S. 2005: "Los depósitos metálicos burgaleses y la metalurgia del Bronce Final en la Meseta Norte: algunas reflexiones". Boletín del Seminario de Estudios de Arte y Arqueología LXXI: 137-59.

Galán Sauliner, C. 1998: "Sobre la cronología de Cogotas I..." Cuadernos de Prehistoria de la Universidad Autónoma de Madrid. 25(1): 201-43.

Garrido Pena, R. 2000: "El Campaniforme en la Meseta Central de la Península Ibérica (c. 2500-2000 a.C.)". BAR International Series 892.

Gener, M.; Rovira LLorens, S.; Montero Ruiz, I. y Rodríguez Vinceiro, F. 2009: "Technological evolu- 
tion of the cutting edges in prehistoric axes and paltstaves". En J.F. Moreau, R. Auger, J. Chabot y A. Herzog (eds.): Proceedings ISA 2006. 36th International Symposium on Archaeometry (2-6 may, 2006, Quebec City, Canada). Quebec, Cahiers d'archéologie du Celat. 25: 179-185.

Gómez Ramos, P. 1993: "Tipología de los lingotes de metal y su hallazgo en los depósitos del Bronce Final de la península ibérica". Cuadernos de Prehistoria de la Universidad Autónoma de Madrid 20: 73-105.

Hayden, B. 1995: "Feasting in prehistoric and traditional societies". En Wiessner, Polly y Schiefenhövel, Wulf (eds.): Food and the status quest. An interdisciplinary perspective. Berghman Books. Providence. Oxford: 127-47.

Herrán Martínez, J.I. 2008: Arqueometalurgia de la Edad del Bronce en Castilla y León. Studia Archaeologica, 95. Universidad de Valladolid. Valladolid.

Jimeno Martínez, A. 1984: Los Tolmos de Caracena, Soria (Campañas de 1977, 1978 y 1979). Nuevas bases para el estudio de la Edad del Bronce en la zona del Alto Duero. Colección Excavaciones Arqueológicas en España, 134. Ministerio de Cultura. Madrid.

Liseau, C.; Blasco, C.; Ríos, P.; Vega, J.; Menduiña, R.; Blanco, J.F.; Baena, J.; Herrera, T.; Petri, A. y Gómez, J.L. 2008: "Un espacio compartido por vivos y muertos: El poblado calcolítico de fosos de Camino de las Yeseras (San Fernando de Henares, Madrid)". Complutum 19 (1): 97-120.

Macarro, J.A. 2002: La Alcalá prehistórica. El poblado de la Edad del Bronce de la Dehesa. Ayuntamiento del Alcalá de Henares. Madrid.

Martín Valls, R. y Delibes de Castro, G. 1989: "La Cultura del Vaso Campaniforme en las campiñas meridionales del Duero: el enterramiento de Fuente-Olmedo". Diputación de Valladolid, Valladolid.

Morán Bardón, C. 1935: Excavaciones en dólmenes de Salamanca y Zamora. Memorias de la Junta Superior del Tesoro artístico, 135. Madrid.
Montero Ruiz, I. 1992: "La actividad metalúrgica en la Edad del Bronce del sudeste de la Península Ibérica: tecnología e interpretación cultural". Trabajos de Prehistoria 49: 189-215.

Montero Ruiz, I. 1993: "Bronze Age metallurgy in southeast Spain". Antiquity 67 (254): 46-57.

Rojo Guerra, M.A.; Kunst, M.; Garrido Pena, R.; García-Martínez de Lagrán, I. y Morán Dauchez, G. 2005: Un desafio a la eternidad: tumbas monumentales en el valle de Ambrona, Arqueología en Castilla y León, 14; Valladolid: Junta de Castilla y León.

Rovira Llorens, S. 2004: “Tecnología metalúrgica y cambio cultural en la Prehistoria de la Península Ibérica". Norba. Revista de Historia 17: 9-40.

Rovira Llorens, S. y Gómez Ramos, P. 1994: "Punzones y varillas metálicas en la Prehistoria Reciente española: un estudio tecnológico". Espacio, Tiempo y Forma. Prehistoria y Arqueología 7: 371-402.

Sherrat, A. 1981: "Plough and pastoralism: aspects of the secondary products revolution". En I. Hodder, G. Isaac y N. Hammond (eds.): Pattern of the Past: Studies in honor of David Clarke. Cambridge University Press. Cambridge: 261-305.

Sherrat, A. 1987: "Cups that cheered". En W.H. Waldren y R.C. Kennard (eds.): Bell Beakers of the Western Mediterranean. Bar International Series 331. Oxford: 81- 103.

Val Recio, J. 1992: "El yacimiento calcolítico precampaniforme de Las Pozas en Casaseca de las Chanas (Zamora)". Boletín del Seminario de Estudios de arte y Arqueología LVIII: 47-62.

Vázquez Cuesta, A. 2009: "Reflexión sobre el campaniforme a partir de la evidencia funeraria de la Cuenca del Tajo (España y Portugal)". Arqueoweb 11.

Vicent García, J.M. 1990: "El Neolitic: tranformacions socials i economiques". En J. Anfruns y E. Llobet (eds.): El canvi cultural a la Prehistòria. Columna. Barcelona: 241-93.

Vicent García, J.M. 1995: "Early social complexity in Iberia: some theoretical remarks". En K.T. Lillios (ed.): The origins of Complex Societies in Late Prehistoric Iberia. Ann Arbor. Michigan. 